\title{
ON THE COMPLETENESS AND THE EXPANSION THEOREM FOR EIGENFUNCTIONS OF THE STURM-LIOUVILLE-ROSSBY TYPE*
}

\author{
BY \\ AKIRA MASUDA \\ University of Tokyo, Tokyo, Japan
}

\begin{abstract}
Rossby waves in a closed ocean form an unfamiliar kind of eigenvalue problem. An investigation is made of the one-dimensional generalized version, which is called here the Sturm-Liouville-Rossby (SLR) problem. The eigenvalue and the eigenfunction of the SLR equation are calculated from those of the associated SturmLiouville (SL) equation and vice versa. The expansion theorem and the completeness theorem for the SLR eigenfunctions are proved to be valid in parallel to the SL problem. Two representations based on the SL eigenfunctions and on the SLR eigenfunctions are provided for Green's function, which gives an example of a reproducing kernel.
\end{abstract}

1. Introduction and formulation. We investigate here an eigenvalue problem, which arises in the study of the dynamic response of the ocean at mid-latitudes. As is well known (Le Blond and Mysak [8]), geophysical fluids are a mine of oscillations and waves originating from various restoring mechanisms such as 1) density stratification (buoyancy force), 2) rotation of the earth (Coriolis force) and 3) the curvature of the earth (beta-effect). Those oscillations and waves are described as eigenfunctions for some forms of differential equations(s). Characteristic frequencies and modes of oscillations have been calculated for a vast variety of oceanographic situations (Le Blond and Mysak [8]) and some mathematical investigations have been made on the spectrum of inertial oscillations in rotating fluids (Franklin [5]; Schaeffer [14]). Nevertheless, the completeness of such geophysical eigenfunctions has rarely been discussed, especially for those associated with the Coriolis force (or the beta-effect). Except for a general conjecture by Greenspan [6], [7], a strict treatment has never been given, so far as the author knows, though some computations and arguments have been published on the basis of this conjecture (e.g., Firing and Beardsley [4]; Miller [13]).

*Received September 7, 1988

(C) 1989 Brown University 
The simplest example of this kind is given by the eigenvalue problem for Rossby normal modes governed by the equation

$$
\begin{cases}\nabla^{2} \psi=-i \lambda \frac{\partial \psi}{\partial x} & \text { in } R \\ \psi=0 & \text { on } \partial R\end{cases}
$$

in a closed rectangular basin defined by $R=(0, \pi) \times(0, Y)$, where $\psi$ is the quasigeostrophic stream function, $(x, y)$ the (eastward, northward) coordinates, $\nabla$ the gradient operator, $\partial R$ the boundary of $R$, nondimensionalization being made so that the basin is $\pi \times Y$ wide. This is reduced to a one-dimensional problem

$$
\frac{d^{2}}{d x^{2}} \varphi(x)-l^{2} \varphi(x)=-i \lambda \frac{d}{d x} \varphi(x),
$$

if we separate the $y$-dependence by putting

$$
\psi(x, y) \sim \varphi(x) \sin l y,
$$

where $l$ denotes the mode number in $y, l Y / \pi$ being an integer in order for the stream function to vanish at the zonal boundaries. The resulting eigenvalues and eigenfunctions of (2) become

$$
\left(\frac{\lambda_{n}}{2}\right)^{2}=l^{2}+n^{2}
$$

and

$$
\varphi_{n}(x)=\frac{2}{\sqrt{\pi}} \frac{1}{\lambda_{n}} \exp \left\{-\frac{i \lambda_{n}}{2} x\right\} \sin n x .
$$

For this set of eigenfunctions, elementary proofs of the completeness and expansion theorem were obtained for the first time, so far as the author knows, in the previous papers (Masuda [11], [12]). By virtue of the theorems it became possible to construct the general linear solution to the initial-value and the response problems of a rectangular closed ocean governed by the quasi-geostrophic equation on a beta-plane; such problems have been dealt with by various methods such as Laplace transformation (Lighthill [9]) and numerical computation based on Chebyshev approximation (Anderson and Gill [1]).

This paper extends the above results to a generalized version of the eigenvalue problem, which is called here the Sturm-Liouville-Rossby problem (for convenience, we adopt an abbreviation 'SLR'). The equation to be considered is

$$
\frac{d}{d x}\left(p(x) \frac{d}{d x} \varphi(x)\right)-q(x) \varphi(x)=-i \lambda \frac{d}{d x} \varphi(x),
$$

or, more generally,

$$
\frac{d}{d x}\left(p(x) \frac{d}{d x} \varphi(x)\right)-q(x) \varphi(x)=-i \lambda\left\{r(x) \frac{d \varphi(x)}{d x}+\frac{d r(x)}{2 d x} \varphi(x)\right\},
$$

with positive $q$ belonging to $C^{0}[0, \pi]$ and positive $p$ and $r$ belonging to $C^{1}[0, \pi], \lambda$ the eigenvalue and $\varphi$ the eigenfunction; the boundary condition is that $\varphi$ be zero at the boundaries. Equation (6) is obtained as a special case of (7) when $r=1$; it is almost the same as the Sturm-Liouville ('SL' for brevity) equation. The important difference is that the eigenvalue is multiplied by the derivative of the eigenfunction 
rather than the eigenfunction itself. Also we must note that $q$ need not be positive in the SL problem. However, we assume it to be positive here, though some relaxation of this restriction is possible; in usual cases of physical significance, $q$ is positive, which assures the eigenvalue of the SL problem is positive (Courant and Hilbert [3]). Hereafter we deal with (7).

2. Eigenvalues and eigenfunctions. Similarly to the prototype equation (2) (Longuet-Higgins [10]), the eigenvalues and eigenfunctions of the SLR problem (7) are related with those of the associated SL problem defined by

$$
\begin{cases}\frac{d}{d x}\left(p(x) \frac{d}{d x} \phi(x)\right)-q(x) \phi(x)=-\mu \frac{r^{2}(x)}{p(x)} \phi(x) & \text { in }(0, \pi) \\ \phi=0 & \text { at } x=0, \pi\end{cases}
$$

where $\mu$ is the eigenvalue and $\phi$ the eigenfunction. It is easy to confirm that

$$
\left(\frac{\lambda_{n}}{2}\right)^{2}=\mu_{n} \quad\left(\lambda_{-n}=-\lambda_{n}\right)
$$

and

$$
\varphi_{n}(x)=\frac{\sqrt{2}}{\lambda_{n}} \exp \left\{\frac{-i \lambda_{n}}{2} \int_{0}^{x} \frac{r(\xi)}{p(\xi)} d \xi\right\} \phi_{n}(x) .
$$

The orthonormal set of the SL equation (8) is obtained immediately as $\left\{\phi_{m} \mid m \in\right.$ $N$ \}, where $N$ denotes the set of positive integers, the eigenfunction $\varphi_{m}$ is chosen as real-valued and normalization is made so that

$$
\left(\phi_{k}, \phi_{l}\right)=\delta_{k, l}
$$

with $\delta_{k, l}$ the Kronecker's delta and the inner product given by

$$
(f, g)=\int_{0}^{\pi} f(x) g^{*}(x) \frac{r^{2}(x)}{p(x)} d x
$$

* being the complex conjugate. In terms of the SL orthonormal eigenfunctions, we have the SLR orthonormal eigenfunctions (10), so that

$$
\left\langle\varphi_{k} \mid \varphi_{l}\right\rangle=\delta_{k, l},
$$

where the inner product is defined by

$$
\langle f \mid g\rangle=\int_{0}^{\pi}\left[p(x) \frac{d f(x)}{d x} \frac{d g^{*}(x)}{d x}+q(x) f(x) g^{*}(x)\right] d x .
$$

The present inner product makes SLR eigenfunctions for different eigenvalues orthogonal to one another. For brevity we call the inner products (12) and (14) as the SL and the SLR inner products, respectively; the corresponding norms become

$$
\|f\|=(f, f)^{1 / 2} \text { and }\langle\langle f\rangle\rangle=\langle f \mid f\rangle^{1 / 2} .
$$

It is to be noted the latter SLR inner norm corresponds to the total (kinetic plus potential) energy of Rossby normal modes. This is a reason why we assume $q$ to be positive. Also a slight manipulation leads to

$$
\left\|\varphi_{n}\right\|^{2}=\frac{2}{\lambda_{n}^{2}}=\frac{1}{2 \mu_{n}}
$$




$$
\begin{gathered}
\left\langle\left\langle\phi_{m}\right\rangle\right\rangle^{2}=\left(\frac{\lambda_{m}}{2}\right)^{2}=\mu_{m}, \\
\left\langle\frac{\varphi_{m}}{\sqrt{\mu_{m}}} \mid \frac{\varphi_{n}}{\sqrt{\mu_{n}}}\right\rangle=\delta_{m, n},
\end{gathered}
$$

and

$$
\left\langle\phi_{m} \mid \varphi_{n}\right\rangle=\mu_{m}\left(\phi_{m}, \varphi_{n}\right) .
$$

Proposition 1. The eigenvalues and eigenfunctions of the SLR equation (7) are obtained by solving the associated SL equation (8) and vice versa; their close relations are expressed by (9), (10), and (16)-(19).

Proposition 2. The SL eigenfunctions make an orthonormal set in the SLR sense, if we adopt $\left\{\varphi_{m} / \sqrt{\mu_{m}}\right\}$ rather than $\left\{\varphi_{m}\right\}$. On the other hand, the SLR eigenfunctions are not orthogonal to one another with respect to the SL inner product.

3. Completeness theorem and expansion theorem. In this section we prove the expansion theorem and the completeness theorem by the use of the theory of compact self-adjoint operators. Suppose that $\widetilde{S}$ is the set of $C^{\infty}$-functions, which have compact supports in $R=(0, \pi)$. The inner product and norm are defined in the previous section; the present norm is equivalent to the Dirichlet norm defined by

$$
\|f\|_{D}=\left\{\int_{0}^{\pi}\left[\left|\frac{d f(x)}{d x}\right|^{2}+|f(x)|^{2}\right] d x\right\}^{1 / 2}
$$

as a metric space. Then, let $S$ denote the Hilbert space as the completion of $\widetilde{S}$ by the SLR norm ( $S$ is identical to Hilbert space $H^{1}$ as a set, on account of the same topology in $S$ and $H^{1}$ ). Any function $f$ in $S$ has its unique weak derivative and has a continuous representation $\hat{f}$, which must vanish at $x=0$ and $\pi$.

Consider a linear operator $L$ which can be defined on $S$ as

$$
\tilde{L}(f)=i \int_{0}^{\pi} K(x, \xi)\left\{r(\xi) \frac{d f(\xi)}{d \xi}+\frac{d r(\xi)}{2 d \xi} f(\xi)\right\} d \xi,
$$

where $K(x, \xi)$ denotes Green's function for the SL differential operator $M_{x}$

$$
M_{x}=\frac{d}{d x}\left(p(x) \frac{d}{d x}\right)-q(x) .
$$

That is, $K(x, \xi)$ satisfies a symbolic equation

$$
M_{x} K(x, \xi)=-\delta(x-\xi),
$$

where $\delta$ denotes Dirac's delta function. The domain of $\tilde{L}$ includes $\widetilde{S}$, which is dense in $S$. If $f$ and $g$ belong to $\widetilde{S}$, we have

$$
\begin{aligned}
\langle f \mid \widetilde{L} g\rangle & =-i \int_{0}^{\pi} f\left(r \frac{d g^{*}}{d x}+\frac{d r}{2 d x} g^{*}\right) d x \\
& =-i \int_{0}^{\pi}\left(r \frac{d f}{d x}+\frac{d r}{2 d x} f\right) g^{*} d x \\
& =\langle\widetilde{L} f \mid g\rangle,
\end{aligned}
$$


which indicates $\widetilde{L}$ to be symmetric. Then, $\widetilde{L}$ is extended to $L$, whose domain is $S$; $L$ is expressed also as (21), with the derivative $d f / d x$ taken as the weak derivative. The continuity of the inner product assures the self-adjointness of $L$ :

$$
\langle f \mid L g\rangle=\langle L f \mid g\rangle
$$

for any $f$ and $g$ belonging to $S$.

Proposition 3. The operator $L$ above is compact.

Proof. Let $\left\{h_{n} \in S \mid n \in N\right\}$ be a sequence of functions whose SLR norms are bounded by a positive number. They are mapped to

$$
f_{n}(x)=i \int_{0}^{\pi} K(x, \xi)\left\{r(\xi) \frac{d h_{n}(\xi)}{d \xi}+\frac{d r(\xi)}{2 d \xi} h_{n}(\xi)\right\} d \xi,
$$

which belong to $C^{1}[0, \pi]$ and vanish at the boundaries. As is well known, Green's function $K(x, \xi)$ is $C^{2}$, except on $x=\xi$, where its first derivative has a finite discontinuity $|1 / p(x)|$. From this smoothness of $K$ and the uniform boundedness of $\left\{h_{n}\right\}$ in $S$, it follows that $\left\{f_{n}\right\}$ and $\left\{d f_{n} / d x\right\}$ are uniformly bounded and equi-continuous as usual functions. The Ascoli-Arzela theorem is applied to guarantee that there is a subset $N^{\prime}$ of $N$, for which subsequences of $\left\{d f_{n} / d x \mid n \in N^{\prime}\right\}$ and $\left\{f_{n} \mid n \in N^{\prime}\right\}$ converge uniformly to continuous function $g_{0}$ and $f_{0}$, respectively. It follows from uniform convergence that the derivative of $f_{0}$ is $g_{0}$ :

$$
\begin{aligned}
f_{0}(x) & =\lim _{n \in N^{\prime}} f_{n}(x)=\lim _{n \in N^{\prime}} \int_{0}^{x} \frac{d f_{n}(\xi)}{d \xi} d \xi=\int_{0}^{x} \lim _{n \in N^{\prime}} \frac{d f_{n}(\xi)}{d \xi} d \xi \\
& =\int_{0}^{x} g_{0}(\xi) d \xi .
\end{aligned}
$$

Hence $f_{0}$ belongs to $C^{1}[0, \pi], f_{0}(0)=f_{0}(\pi)=0$ and $d f_{0} / d x=g_{0}$. This means that $f_{0}$ belongs to $S$, which in turn implies $L$ to be compact.

According to the theory of compact self-adjoint operators (for example, Bachman and Narici [2]), the eigenfunctions of operator $L$ make a complete orthonormal basis of space $S$. It is easy to confirm that an eigenfunction $\varphi_{n}$ of the integro-differential operator $L,(21)$, is also an eigenfunction of the differential eigenvalue equation (7), if we use the fact that any $f$ belonging to $S$ has a continuous representation vanishing at $x=0$ and $\pi$. Note that the eigenvalue of $L$ is the inverse of that for (7):

$$
L\left(\varphi_{n}\right)=\frac{-i}{\lambda_{n}} \varphi_{n}
$$

This completes the proof of the following theorem.

CoMPLETENesS THeOREM. The orthonormal set composed of eigenfunctions of (7) is complete in $S$; in $H^{1}$, it is not orthonormal, but complete.

A stronger theorem is derived immediately.

Expansion Theorem. If a function $f$ belongs to $S$, it can be expanded in a series in the eigenfunctions $\varphi_{n}$; this series converges uniformly and absolutely.

Proof. The formal Fourier expansion of $f$ is given by

$$
f(x) \sim \sum_{n}^{\prime}\left\langle f \mid \varphi_{n}\right\rangle \varphi_{n}(x)
$$


where $\sum_{n}^{\prime}$ denotes the summation over integers $n$ except for zero. Bessel's inequality assures that

$$
\langle\langle f\rangle\rangle^{2} \geq \sum_{n}^{\prime}\left|\left\langle f \| \varphi_{n}\right\rangle\right|^{2}
$$

Likewise we have

$$
\langle\langle K(x, \xi)\rangle\rangle_{\xi}^{2} \geq \sum_{n}^{\prime}\left|\left\langle K \mid \varphi_{n}\right\rangle_{\xi}\right|^{2},
$$

where the suffix $\xi$ denotes the operation with respect to $\xi$. Since $\varphi_{n}$ is an eigenfunction satisfying (7) and (28), we have

$$
\left\langle K \mid \varphi_{n}\right\rangle_{\xi}=\varphi_{n}(x) .
$$

Using the Schwarz inequality for the summation (28) and applying (30)-(32), we obtain

$$
\left\{\sum_{n}^{\prime}\left|\left\langle f \mid \varphi_{n}\right\rangle \varphi_{n}(x)\right|\right\}^{2} \leq\langle\langle f\rangle\rangle^{2} \max _{x \in[0, \pi]}\langle\langle K\rangle\rangle_{\xi}^{2} .
$$

The right-hand side is independent of $x$, so that the formal Fourier series converges uniformly and absolutely to a certain continuous function $\tilde{f}$. The function $f-\tilde{f}$ is orthogonal to any eigenfunction in the SLR sense. Therefore the completeness theorem assures that $f-\tilde{f}=0$ in space $S$.

COMPLETENESS THEOREM IN $L^{2}$. The orthonormal sets of the eigenfunctions for the SLR problem are orthonormal neither in the SL sense nor in the $L^{2}$ sense, in general. Still they are complete both in the SL and in the $L^{2}$ sense.

Proof. Obviously any $L^{2}$ function can be approximated arbitrarily well by a certain $C^{1}$ function which vanishes at boundaries $x=0$ and $\pi$. In turn, such a $C^{1}$ function can be approximated arbitrarily well by a linear combination of the SLR eigenfunctions in the SLR norm. Since the SLR norm is stronger than the $L^{2}$ and the SL norm (Poincaré's inequality [3]), $f$ can be approximated by a linear combination of the SLR eigenfunctions.

4. An alternative elementary proof, transformation laws and the reproducing kernel. This section presents an elementary proof without appealing to the theory of compact operators, and describes relations between the SL and the SLR norms and between the SL eigenfunctions and the SLR eigenfunctions. The proof here can be extended to singular cases when $p(x)$ vanishes at boundaries. An example is provided in the Appendix.

LEMma. The SL eigenfunctions are approximated arbitrarily well in terms of the SLR eigenfunctions both in the SL and in the SLR metrics.

Proof. Since the SL eigenfunctions $\left\{\varphi_{m} \mid m \in N\right\}$ are complete with respect to the SL norm, each of the SLR eigenfunctions $\varphi_{n}$ can be expanded in terms of the eigenfunctions of the former:

$$
\varphi_{n}(x)=\sum_{m=1}^{\infty}\left(\varphi_{n}, \phi_{m}\right) \phi_{m}(x) .
$$


Parseval's identity gives for each $n$

$$
\left\|\varphi_{n}\right\|^{2}=\sum_{m=1}^{\infty}\left|\left(\varphi_{n}, \phi_{m}\right)\right|^{2} .
$$

In the same way the SL eigenfunction has its formal Fourier series with respect to the SLR eigenfunctions as

$$
\phi_{m}(x) \sim \sum_{n}^{\prime}\left\langle\phi_{m} \mid \varphi_{n}\right\rangle \varphi_{n}(x) .
$$

Bessel's inequality for the SLR norm yields

$$
\left\langle\left\langle\phi_{m}\right\rangle\right\rangle^{2} \geq \sum_{n}^{\prime}\left|\left\langle\phi_{m} \mid \varphi_{n}\right\rangle\right|^{2}
$$

which becomes

$$
\frac{1}{\mu_{n}} \geq \sum_{n}^{\prime}\left|\left(\phi_{m}, \varphi_{n}\right)\right|^{2}
$$

by virtue of (17) and (19).

Now let us consider a positive double series

$$
Q=\sum_{m=1}^{\infty} \sum_{n}^{\prime}\left|\left(\phi_{m}, \varphi_{n}\right)\right|^{2} .
$$

According to the SL theory (Courant and Hilbert [3]), the asymptotic behavior of the SL eigenvalue becomes

$$
\lim _{m \rightarrow \infty} \frac{m^{2}}{\mu_{m}}=\frac{1}{\pi^{2}} \int_{0}^{\pi} \frac{r(x)}{p(x)} d x
$$

which allows a majorant with a positive constant $C$ :

$$
\frac{1}{\mu_{m}} \leq C \frac{1}{m^{2}} \text {. }
$$

Hence the positive summation (39) converges to a certain value. Inequality (38) together with (29) yields

$$
\sum_{m=1}^{\infty} \frac{1}{\mu_{m}} \geq Q=\sum_{n=1}^{\infty} \frac{1}{\mu_{n}}
$$

which leads to the identity for each $m$ :

$$
\frac{1}{\mu_{m}}=\sum_{n}^{\prime}\left|\left(\phi_{m}, \varphi_{n}\right)\right|^{2}
$$

Rewriting (43) with the use of (17) and (19) as

$$
\left\langle\left\langle\phi_{m}\right\rangle\right\rangle^{2}=\sum_{n}^{\prime}\left|\left\langle\phi_{m} \mid \varphi_{n}\right\rangle\right|^{2}
$$

we conclude that an adequate series in terms of the SLR eigenfunctions can approximate any SL eigenfunctions in the SLR metric. Applying Poincaré's inequality (Courant and Hilbert [3]) with a slight modification

$$
\|q\|^{2} \leq D\langle\langle q\rangle\rangle^{2}
$$


for a certain positive $D$ and any $C^{1}$ funct: $\mathrm{n} q(x)$ vanishing at the boundaries $x=0$ and $\pi$ (or any function in $S$ ), we have

$$
\lim _{N \rightarrow \infty}\left\|\phi_{n}-\sum_{n=-N}^{N}\left\langle\phi_{m} \mid \varphi_{n}\right\rangle \varphi_{n}\right\|=0 .
$$

Since the set of the SL eigenfunctions is complete for the SL norm, so is the set of the SLR eigenfunctions, though they are not orthogonal to one another with respect to the SL metric.

Transformation laws and the expansion theorem follow from the above lemma.

Proposition 4. For $q \in C^{1}[0, \pi]$ and vanishing at the boundaries (or $q \in S$ ), the following equations hold:

$$
\begin{aligned}
& \left(q, \phi_{m}\right)=\sum_{n}^{\prime}\left\langle q \mid \varphi_{n}\right\rangle\left(\varphi_{n}, \phi_{m}\right) \\
& \delta_{k, m}=\sum_{n}^{\prime}\left\langle\phi_{k} \mid \varphi_{n}\right\rangle\left(\varphi_{n}, \phi_{m}\right) \\
& \left\langle q \mid \varphi_{n}\right\rangle=\sum_{m=1}^{\infty}\left(q, \phi_{m}\right)\left\langle\phi_{m} \mid \varphi_{n}\right\rangle \\
& \delta_{k, n}=\sum_{m=1}^{\infty}\left(\varphi_{k}, \phi_{m}\right)\left\langle\phi_{m} \mid \varphi_{n}\right\rangle \\
& \|q\|^{2}=\sum_{m=1}^{\infty}\left|\left(q, \phi_{m}\right)\right|^{2} \\
& \langle\langle q\rangle\rangle^{2}=\sum_{m=1}^{\infty}\left|\left\langle q \mid \frac{\phi_{m}}{\sqrt{\mu_{m}}}\right\rangle\right|^{2}=\sum_{n}^{\prime}\left|\left\langle q \mid \varphi_{n}\right\rangle\right|^{2} \\
& q(x)=\sum_{m=1}^{\infty}\left(q, \phi_{m}\right) \phi_{m}(x) \\
& q(x)=\sum_{m=1}^{\infty}\left\langle q \mid \frac{\phi_{m}}{\sqrt{\mu_{m}}}\right\rangle \frac{\phi_{m}(x)}{\sqrt{\mu_{m}}}=\sum_{n}^{\prime}\left\langle q \mid \varphi_{n}\right\rangle \varphi_{n}(x) .
\end{aligned}
$$

In particular, (52) means that the SL eigenfunctions are not only orthonormal (Proposition 2), but also complete in the SLR metric. The series in (53) and (54) converge uniformly and absolutely (expansion theorem).

Proof. The proof proceeds just in the same way as in Masuda [12]. Therefore we omit the details and merely describe the outline. Using the lemma, we can derive transformation laws (47)-(50). A formal Fourier series of $q$ in terms of $\varphi_{n}(54)$ is shown to converge uniformly and absolutely to a continuous function $\tilde{q}$ with the aid of (33). The Fourier coefficients of $q$ and $\widetilde{q}$ with respect to $\phi_{m}$ are equal to each other by virtue of transformation laws. Consequently $q$ is identical to $\widetilde{q}$, which proves the required expansion theorem. For any function in $\widetilde{S}$, Parseval's identities are valid owing to the uniform convergence of the expansion in terms of $\varphi_{n}(54)$. Since $S$ is the completion of $\widetilde{S}$, the same identities do hold on $S$. 
Note that (54) are expressed formally as

$$
\begin{aligned}
q(x) & =\left\langle q(\xi) \mid \sum_{m=1}^{\infty} \frac{\phi_{m}(\xi) \phi_{m}(x)}{\sqrt{\mu_{m}} \sqrt{\mu_{m}}}\right\rangle_{\xi} \\
& =\left\langle q(\xi) \mid \sum_{n}^{\prime} \varphi_{n}(\xi) \varphi_{n}^{*}(x)\right\rangle_{\xi},
\end{aligned}
$$

which give two representations of the reproducing kernel $N(x, \xi)$ :

$$
\begin{aligned}
N(x, \xi) & =\sum_{m=1}^{\infty} \frac{\phi_{m}(x) \phi_{m}(\xi)}{\mu_{m}} \\
& =\sum_{n}^{\prime} \varphi_{n}(x) \varphi_{n}^{*}(\xi) .
\end{aligned}
$$

If we rewrite (55) or (56) as

$$
q(x)=\langle N(x, \xi) \mid q(\xi)\rangle_{\xi}=-\int_{0}^{\pi} N(x, \xi) M_{\xi} q(\xi) d \xi,
$$

$N(x, \xi)$ turns out to be $K(x, \xi)$, Green's function for $M_{x}$ in the previous section. The above results are summarized as follows.

THEOREM. Green's function $K(x, \xi)$ for the SL differential operator $M_{x}$ is the reproducing kernel of Hilbert space $S\left(H^{1}\right)$; it has two representations (57) and (58) based on the SL eigenfunctions (Marcer's theorem [3]) and on the SLR eigenfunctions, respectively.

Finally in this section, let us remark that the elementary method of proof in Masuda [11] can be applied to the present generalized case as well with minor modifications, if we use the asymptotic behavior of eigenvalues (40) and the relations are clarified between the SL eigenfunctions and the SLR eigenfunctions mentioned in Sec. 2. For that purpose, we first note that the reproducing kernel (Green's function) is represented by (57) (Marcer's theorem [3]). Then, it is shown to be expressed as (58) by considering the SLR norm of the difference between the two representations. This identity (58) leads to the expansion theorem straightforwardly. The completeness theorem is an almost trivial consequence. See Masuda [11] for this way of proof.

5. Discussion. In this paper, the Sturm-Liouville-Rossby eigenvalue problem is introduced as an extension of the reduced one-dimensional problem for Rossby normal modes in a rectangular basin on a beta-plane. The expansion theorem and the completeness theorem are proved in parallel to the usual Sturm-Liouville problem. The relations between eigenfunctions of the SLR problem and those of the associated SL problem are clarified; in particular, any orthonormal system of usual SL special functions is found to be accompanied by the associated SLR orthonormal set and vice versa.

We may say, however, that completeness problems adequate for geophysical fluids still remain almost completely unexplored. It is desirable to solve the completeness 
problem because 1) the possible motion resolves into a superposition of normal oscillations, the property of which is well understood and consequently 2) the general linear solution is obtained immediately. If normal modes are not complete on the contrary, which is unlikely, it is of great interest and significance to find what kind of motion should supplement normal modes.

When the basin is not rectangular but arbitrary, the problem cannot be reduced to a one-dimensional case. We must deal with two-dimensional problems, where Green's function has a logarithmic singularity, which makes it impossible to apply such a simple argument as in the present paper. For example, the left-hand side of (31), which has been used for the proof of the expansion theorem, diverges to infinity. This case will be discussed on a future occasion.

As explained in the introduction, the completeness of the present type of eigenvalue problem cannot be reduced to the usual completeness theory of the SL type. In the real ocean dynamics, further complicated forms of eigenvalue problems appear for different situations. Even if we restrict ourselves to the quasi-geostrophic regime, the density stratification and uneven bottom topography inevitably make the problem three-dimensional. Probably more difficult will be the case without quasi-geostrophic assumption. As the simplest situation, consider the following equation in a twodimensional basin $R$ :

$$
\left\{\begin{array}{l}
\frac{\partial u}{\partial t}-f v=-\frac{1}{\rho} \frac{\partial p}{\partial x} \\
\frac{\partial v}{\partial t}+f u=-\frac{1}{\rho} \frac{\partial p}{\partial y} \\
\frac{1}{\rho g} \frac{\partial p}{\partial t}+\frac{\partial}{\partial x}(H u)+\frac{\partial}{\partial y}(H v)=0
\end{array}\right.
$$

where $f$ denotes the Coriolis parameter and $H(>0)$ the depth, the others being usual notations; the normal velocity vanishes at the boundaries. This produces an eigenvalue problem as well, where normal oscillations should have mixed properties of inertial-gravity waves, Kelvin waves, and shelf waves (Miller [13]). For this situation, there may occur geostrophic flows with zero eigenvalue; the eigenspace associated with geostrophic flows has an infinite dimension. Are vector-valued eigenfunctions $\left\{u_{n}, v_{n}, p_{n}\right\}$ complete? In that case, what will be the definition of the completeness? So far as the author knows, no explicit studies have been published on this kind of problem, except for the general conjecture of Greenspan [6], [7]. The completeness problem is one of the most unsettled subjects in the field of long-period waves in geophysical fluids. The present investigation including Masuda [11], [12] is probably the first (I hope) that gives a strict result, though it touches only a part of the vast area.

Acknowledgment. I would like to thank Prof. K. Kajiura for his encouraging discussion, Dr. R. Greatbatch for correcting grammatical errors, and Ms. M. Hojo for preparing the manuscript.

Appendix. SLR orthonormal sets associated with Bessel functions. Let us give an example of the SLR eigenfunctions with $p(x)$ vanishing at a boundary. For a given 
order $k$, the first kind of Bessel functions $\left\{J_{k}\left(\sqrt{\mu_{k, m}} x\right) \mid m \in N\right\}$ form a complete set in the SL sense (Courant and Hilbert [3]), where $\sqrt{\mu_{k, m}}$ denotes the $m$ th zero of $J_{k}(x)$. The SL orthonormal functions become

$$
\phi_{m(x)}=\frac{\sqrt{2}}{J_{k}^{\prime}\left(\sqrt{\mu_{k, m}}\right)} J_{k}\left(\sqrt{\mu_{k, m}} x\right) .
$$

where the prime indicates the derivative. The associated SLR eigenfunction is calculated to be

$$
\varphi_{n}(x)=\frac{1}{\sqrt{\mu_{k, n}} J_{k}^{\prime}\left(\sqrt{\mu_{k, n}}\right)} \exp \left\{-i \sqrt{\mu_{k, n}} x\right\} J_{k}\left(\sqrt{\mu_{k, n}} x\right)
$$

for positive $n$ and the complex conjugates for negative $n$. The SLR eigenfunction $\varphi_{n}(x)$ is regular at $x=0$, vanishes at $x=1$, and satisfies the differential equation

$$
\frac{d}{d x}\left(x \frac{d \varphi_{n}}{d x}\right)-\frac{k^{2}}{x} \varphi_{n}=-2 i \sqrt{\mu_{k, n}}\left(x \frac{d \varphi_{n}}{d x}+\frac{1}{2} \varphi_{n}\right) .
$$

The completeness of the SLR eigenfunctions are proved as in Sec. 4; since $\mu_{k, m}$ is of the order of $m^{2}$ for large $m,(51)$ does hold for this singular case.

Note that when $k=0$, the second term of the left-hand side of $(63)$ is zero. Consequently, we have an orthonormal complete set in the SL sense composed of

$$
\frac{d \varphi_{n}}{d x}=\frac{J_{1}\left(\sqrt{\mu_{0, n}} x\right)+i J_{0}\left(\sqrt{\mu_{0, n}} x\right)}{J_{1}\left(\sqrt{\mu_{0, n}}\right)} \exp \left\{-i \sqrt{\mu_{0, n}} x\right\} .
$$

\section{REFERENCES}

[1] D. L. T. Anderson and A. E. Gill, Spin-up of a stratified ocean, with application to upwelling, Deep-Sea Research 22, 583 (1975)

[2] G. Bachman and L. Narici, Functional Analysis, Academic, 1966

[3] R. Courant and D. Hilbert, Methods of Mathematical Physics, Interscience, Vol. 1, 1953, Vol. 2, 1962

[4] E. Firing and R. C. Beardsley, The behavior of a barotropic eddy on a beta-plane, J. Phys. Oceanogr. 6, 67 (1976)

[5] J. E. Franklin, Axisymmetric inertial oscillations of a rotating fluid, J. Math. Anal. Appl. 39, 742 (1972)

[6] H. P. Greenspan, On the transient motion of a contained rotating fluid, J. Fluid Mech. 20, 673 (1964)

[7] H. P. Greenspan, On the general theory of contained rotating fluid motions, J. Fluid Mech. 22, 449 (1965)

[8] P. H. LeBlond and L. A. Mysak, Waves in the Ocean, Elsevier, 1978

[9] M. J. Lighthill, Dynamic response of the Indian ocean to the onset of the south west monsoon, Phil. Trans. Roy. Soc. London 265, 45 (1969)

[10] M. S. Longuet-Higgins, Planetary waves on a rotating sphere, Proc. Roy. Soc. London A 279, 446 (1964)

[11] A. Masuda, $A$ proof and applications of the expansion theorem for the Rossby normal modes in a closed rectangular basin, J. Oceanogr. Soc. Japan 43, 237 (1987)

[12] A Masuda, A supplementary note on the completeness of the Rossby normal modes in a rectangular basin, J. Oceanogr. Soc. Japan 44, 40 (1988)

[13] A. J. Miller, Nondivergent planetary oscillations in midlatitude ocean basins with continental shelves, J. Phys. Oceanogr. 16, 1914 (1986)

[14] D. G. Schaffer, On the existence of discrete frequencies of oscillation in a rotating fluid, Stud. Appl. Math. 54, 269 (1975) 